Coppen, A., Chaudhry, S. \& Swade, C. (1986) Folic acid enhances lithium prophylaxis. Journal of Affective Disorders, 10, 9-13.

Godfrey, P. S. A., TOONE, B. K., CARNeY, M. W. P., et al (1990) Enhancement of recovery from psychiatricillness by methylfolate. Lancet, 336, 392-395.

LeE, S., Chow, C. C., Shek, C. C., et al (1992) Folate concentration in Chinese psychiatric outpatients on longterm lithium treatment. Journal of Affective Disorders (in press).

Procter, A. (1991) Enhancement of recovery from psychiatric illness by methylfolate. British Journal of Psychiatry, 159, 271-272.

YUN KWOK WING

SING LEE

Prince of Wales Hospital

Chinese University of Hong Kong

Hong Kong

\section{Christmas disease and major affective disorder}

SIR: We were interested to read Gill et al's description of a pedigree cosegregating Christmas disease and major affective disorder (Journal, January 1992, 160, 112-114). We would like to report a further family in which both disorders are segregating.

The proband, a 44-year-old man, has laboratoryproven Christmas disease and DSM-III-R bipolar disorder. His 40-year-old sister is a carrier of the Christmas disease gene and has DSM-III-R lifetime diagnoses of major depression and alcohol abuse. Her eldest son, who is 19 years old, has laboratoryproven Christmas disease and DSM-III-R major depression and alcohol abuse. The proband's father, who is 67 years old, is haematologically normal but had a single episode of DSM-III-R major depression at the age of 47 years. The proband's mother, who is 68 years old, is a carrier of the Christmas disease gene and had a two-year episode of depression at the age of 30 years which necessitated psychiatric referral but which only satisfies DSM-III-R criteria for depression, not otherwise specified. The proband has a haematologically normal son and four daughters who are obligate carriers of the Christmas gene. The proband's sister has two other sons in addition to the one already described: one who has Christmas disease and the other who is haematologically normal. None of these seven children has expressed affective disorder but all are below the age of 18 years and, thus, they are only starting to approach the risk period.

This pedigree is consistent with genetic linkage between the Christmas disease gene and a susceptibility locus for affective disorder but does not offer strong support for this hypothesis because of: (a) the small size of the pedigree, and (b) complications in interpretation introduced by assortative mating between the proband's parents. Like Dr Gill et al we examined the proband's karotype and performed molecular genetic studies on the proband's DNA in the region of the factor IX gene and found no evidence of any abnormality. Thus, it is unlikely that a single mutation such as a large deletion caused both disorders. Clearly our small pedigree may have arisen by chance but like several other pedigrees in the literature it focuses interest on the possibility of an affective disorder susceptibility locus in the $\mathrm{Xq} 27-28$ region.

Assuming no population association between Christmas disease and bipolar disorder, we estimate that there should be at least five men with both disorders in the United Kingdom. Drs Gill et al have reported one and we have reported another. Perhaps investigation of the families of some of the other doubly affected probands may further inform the continuing debate about the existence of $\mathrm{X}$-linked susceptibility loci in affective disorder (Hebebrand, 1992).

Hebebrand, J. (1992) A critical appraisal of X-linked bipolar illness. Evidence for the assumed mode of inheritance is lacking. British Journal of Psychiatry, 160, 7-11.

Nick Craddock

University of Birmingham

Queen Elizabeth Psychiatric Hospital

Birmingham B15 2QZ

Department of Psychological Medicine and

Mike OWEN

Institute of Medical Genetics

University of Wales College of Medicine

Heath Park

Cardiff CF4 $4 X N$

\section{Survivors of disaster}

SIR: Dr Plummer's critique (Journal, March 1992, $160,420-421$ ) of our study of causal attributions and psychiatric symptoms in survivors of the Herald of Free Enterprise disaster (Journal, October 1991, 159, 542-546) is based on a misunderstanding of our work and on misapplications of statistical principles.

He begins, for example, by listing a number of "obvious methodological problems". Our sample, which we described carefully and in some detail, was certainly small and unrepresentative, but it is not clear how this fact alone could account for the pattern of findings. He then reproaches us for using correlation rather than regression analysis, omitting to mention that we used partial correlation analysis (regression is based on partial correlations). This confusion between correlations and partial correlations may also account for his puzzling charge that we had 
assumed "that correlation implies causation". Our use of partial correlations to test the ability of attributional scores to predict later symptoms, controlling for the effect of prior symptoms, is a well validated technique of causal modelling. We did report first-order Pearson correlations since the partial correlations were based on these, but readers will be reassured to learn that the pattern of findings with non-parametric correlations was essentially identical.

Dr Plummer then complains that we "started with nominal data (e.g. the categories 'internal', 'external' and 'both internal and external'), which they arbitrarily convert to ordinal data (e.g. categories ' 1 , ' 2 ' and ' 1.5 '). This is then processed to give discrete (discontinuous) numerical data". But the 'internal' and 'external' categories are at opposite ends of a prespecified attributional dimension and represent one of the commonest oppositions in social psychology. It is inappropriate to describe them simply as "nominal data', a term more commonly used to refer to such variables as religious affiliation (Catholic, Protestant, etc.) which do not stand in any recognised relationship to each other.

He points out, correctly, that the scores subjects can obtain are constrained by the number of attributional statements they make. But it does not follow from this that the data cannot be analysed by methods appropriate to continuous numerical data, provided that the distribution approximates to a continuous distribution and the basic assumptions of interval scaling are met. Another important point is that each subject can in principle obtain any score in the range $1-2$, to at least one place of decimals. In practice the possible scores are limited to some extent by the number of event statements. But there is no reason to think that the number of event statements is systematically related to our outcome variables.

Dr Plummer next tries to argue, using the binomial theorem, that when the average internality or controllability rating is near to one of its extreme values, subjects with larger numbers of event statements have a vanishingly small probability of obtaining certain scores compared with subjects with few event statements. But this claim is based on a misapplication of the binomial theorem. To begin with, subjects in our study could obtain one of three possible scores, not one of two as required by the theorem. Secondly, the theorem assumes that all the outcomes are independent events. In fact the attributional statements were not at all independent and the use of the binomial theorem is therefore invalidated. We have nevertheless calculated the correlations between the number of event statements on which subjects' scores were based and their score on the two attributional dimensions. The correlation with controllability was -0.18 and with internality 0.31 - neither correlation was significant.

We do not wish to suggest that this particular piece of research is beyond criticism - far from it. We are well aware of the limitations of what is essentially a pilot study and did our best to draw attention to its limitations in our report. Dr Plummer's criticisms, however, do not seem to us likely to improve understanding of the difficulties of doing research in this particular area. We hope he and other readers will be reassured by the fact that our basic findings have now been partially replicated in another sample (Joseph et al, 1992).

We are grateful to Geoff Der for generous statistical advice. Joseph, S. A., Brewin, C. R.. YuLE, W., et al (1992) Causal attri-
butions and post-traumatic stress in children. Journal of Child
Psychology and Psychiairy (in press).

$M R C$ Social and Community Psychiatry Unit

Steve A. Joseph

CHRIS R. BREWIN

William Yule

RUTH Williams

Institute of Psychiatry

De Crespigny Park

London SE5 8 AF

Adrenocortical suppression presenting with agitated depression, morbid jealousy, and a dementia-like state

SIR: In response to Professor Cohen's letter (Journal, April 1992, 160, 566), blood sugar measures were carried out during our patient's illness, on three occasions, and were reported as being in the normal reference range ( 3.3 to $5.5 \mathrm{mmol} / \mathrm{l}$ ).

At a clinical level, our patient's symptoms were also not consistent with a hypoglycaemic state: they were constant rather than episodic and were unrelated to meals.

With regard to restoration of endocrine function in place of lifelong treatment with prednisolone, advice from an endocrinological colleague was that as our patient (now aged 69 years) benefited from prednisolone both for her asthmatic disorder and her neuropsychiatric state, maintenance treatment with prednisolone was justified on a longterm basis.

\section{St Nicholas Hospital}

F. HASSANYEH

\section{Jubilee Road}

Gosforth

Newcastle upon Tyne NE3 $3 X T$ 